\title{
Stratégies des planteurs de cocotiers au Ghana face à la maladie du jaunissement mortel
}

\author{
François RUF ${ }^{1}$ \\ Serge BINI ${ }^{2}$ \\ Kwame AMPADU² \\ ${ }^{1}$ CIRAD, UMR Innovations \\ <francois.ruf@cirad.fr> \\ ${ }^{2}$ ONG « Agriculture et Cycle de vie »
}

\begin{abstract}
In Chana, coconut developed extensively during the 1960s, 1970s and 1980s in the coastal strip of the "Western" region. Subsequently, many coconut plantations disappeared as they were progressively affected by lethal yellowing disease. The response of the planters was to stop investing in coconut, then, after an interval of a few years, to start diversifying by planting oil palm, cocoa and rubber. At the same time, villages located further inland, which were untouched by the disease, continued to produce coconut. Between these two areas, in the "heart" of the coconut production region around Jomoro, coconut plantations were not affected by the disease, but were aging and undergoing a decrease in yield. The result of these developments was the relocation of production centres, which is the subject of analysis in this paper. We used a model that was developed for other perennial crops in conditions of almost complete monoculture, particularly cocoa. The model revealed a pattern of regional cycles succeeding one another that are relatively independent of changes in coconut prices. The cycles are based on the exhaustion of natural resources as a source of income, and on the concurrence of the life cycle of the plantation and that of family life. The model was first successfully tested on the situation of coconut in Ghana before 2005-2006. In 2005-2006, a major economic upheaval occurred. The arrival of Nigerian buyers helped cause a $50 \%$ increase in the price of coconut. Prices continued to rise in 2007. From a strictly technical and economic point of view, given the prices offered by Nigerian buyers, the cultivation of hybrid coconut, which is less vulnerable than the local coconut, again appeared to be very profitable, with a risk factor that was acceptable in regions not affected by the disease. These economic conditions enabled us to check what the model would predict in the case of a rise in prices, which was that in coconut production areas, the response would be very limited, i.e. the opposite of what classical theory predicts. In fact, in areas of aging monocrops, a rise in prices no longer has much effect. The model explained the real state of affairs at the regional scale: the recession of the previously leading crop is almost inevitable. On the other hand, a favourable response to the rise in prices is possible in districts and provinces in Chana (and in Côte d'Ivoire) that have not been affected by the disease, thus reinforcing the process of relocation of production centres. This structural relocation could mean a new future for coconut in Chana in the context of expanding regional demand in Nigeria, Burkina Faso and Mali. Yet the end of research, the end of the production of planting material, the lack of low-interest credit, (a precondition for re-investment), and finally, the abandonment of coconut planters by public policies, has left thousands of families and particularly old people, trapped in a spiral of recession which the rise in the price of coconut will not break.
\end{abstract}

Key words: price response, economic cycle, life cycle, monoculture, diversification, indebtedness
Au Ghana, le cocotier connaît un fort développement dans les années 1960 et surtout 1970 et 1980, dans la région Ouest, du moins sur sa frange côtière [1, 2]. Puis, progressivement touchées par la maladie du jaunissement mortel, de nombreuses plantations disparaissent. Entre 1999 et 2003, un projet financé par l'AFD (Agence française de développement), tente de consolider le secteur en favorisant l'adoption d'un hybride, supposé tolérant à la maladie.
Mais, dès 2004, les résultats s'annoncent médiocres. Le taux d'adoption des hybrides est faible. Les planteurs ont sans doute été sélectionnés trop rapidement [3]. Surtout, appauvris par la mortalité de leurs vieux cocotiers et par les prix médiocres offerts par la filière locale $d^{\prime}$ 'huile de coco, les planteurs n'ont guère les moyens d'investir et d'entretenir les cocotiers hybrides. En 2004, presque un tiers des plantations d'hybrides sont en voie de quasi-abandon [4].
Dans les villages touchés par la maladie, la seule stratégie possible semble la diversification vers le palmier, le cacaoyer et l'hévéa, du moins dans les régions à pluviométrie abondante comme la région d'Axim [1, 2]. L'avenir de la filière cocotier semble alors bien compromis, au moins dans le sud de cette région Western, la « boucle » du cocotier au Chana.

En 2005-2006, survient un changement économique majeur. L'arrivée d'acheteurs nigé- 
rians contribue à faire doubler les prix des noix de coco [5, 6]. En 2007, le prix continue de monter. Ce bouleversement est-il de nature à sauver la filière cocotier dans la région et plus largement au Ghana? Sur un plan strictement technico-économique, malgré les risques liés à la maladie, la réponse serait plutôt positive. La culture du cocotier hybride, conduite sur un mode semi-intensif ou intensif pourrait être rentable (voir la communication de Serge Bene, dans ce numéro).

Néanmoins, selon un modèle construit et testé empiriquement, notamment pour les économies cacaoyères, dans un contexte de quasimonoculture et de vieillissement des plantations et des planteurs, la capacité de réponse des exploitations familiales à une hausse de prix est très émoussée. Le modèle montre que la récession de la culture, autrefois dominante, est quasi inévitable. En revanche, la réponse favorable à la hausse de prix peut s'exprimer dans de nouvelles régions, engendrant un déplacement des foyers de production. Ce déplacement serait donc structurel. L'objet de cette étude est de tester l'hypothèse et le modèle sur ce cas d'école : face à une hausse brutale du prix, comment réagit une économie basée sur un verger en quasi-monoculture, dominant les paysages, mais un verger vieillissant, aux faibles rendements? Relance ou récession?

\section{Modèle qualitatif des cycles de matières premières agricoles}

Le modèle a été construit par comparaison de fronts pionniers cacaoyers, en Côte-d'Ivoire, au Ghana, en Amérique du Sud, en Asie du SudEst. Des premiers booms du cacao en Amérique centrale au $x v l^{e}$ siècle jusqu'au plus récent de I'Indonésie, nous avons observé une grande récurrence des mécanismes en œuvre [7-11].

\section{Usure des terres}

En règle générale, la nouvelle culture profite $d^{\prime}$ 'un milieu vierge, d'une rente forêt ou plus largement d'une rente «milieu naturel ». Une rente forêt existe chaque fois qu'il est plus cher de replanter et de remplacer les mêmes arbres sur une même parcelle que d'aller les planter en défrichant une parcelle de forêt. La majorité des cultures dites pérennes, telles que cacaoyer, caféier ou poivrier, réussissent très bien après une défriche/brûlis de forêt. Dès que les conditions d'un marché se créent, la nouvelle culture attire les producteurs, souvent des migrants, avides de revenus et de terres. La production et les revenus augmentent rapidement dans la région forestière (figure 1). de richesse devient source de problèmes. Un changement écologique s'opère, la perte de matière organique dans le sol augmente les risques de mortalité. Des insectes ou autres pestes s'adaptent à la culture, laminent sa rentabilité. Les raisons du développement des insectes et de certaines maladies sont à la fois relatives à la disparition de la forêt (perte de la rente forêt) et aux externalités de la monoculture (perte de la biodiversité, risques accrus liés à la concentration d'une culture dans un espace donné). Les causes peuvent être indirectes, tel un changement de microclimat favorisant la sécheresse, les risques d'incendie ou un dégât quelconque. Les causes peuvent être plus directes. Par exemple, si un insecte utilise un arbre de la forêt naturelle comme hôte privilégié ou
Puis, quelque 20 ou 30 ans plus tard, la source

comme source de nourriture, sa disparition va amener l'insecte à se reporter sur la culture qui remplace la forêt.

Dans le cas du cacao, même si les cas ne sont pas toujours clairement démontrés, c'est probablement ce qui explique les développements $d^{\prime}$ attaques de foreurs de tiges (larves d'insectes) et de termites, dans plusieurs régions du monde, tels que le foreur de cabosses en Asie du Sud-Est. Dans tous les cas, la replantation est rendue difficile par une croissance lente des arbres et un taux élevé de mortalité $[12,13]$. Avant l'échéance de la replantation, I'arbre/la culture pérenne grandit, ce qui rend la récolte plus pénible, avec des fruits plus haut perchés (problèmes fréquemment cités par les planteurs de cacaoyers mais aussi et surtout de palmiers). D'une façon générale, au bout de 20 ou

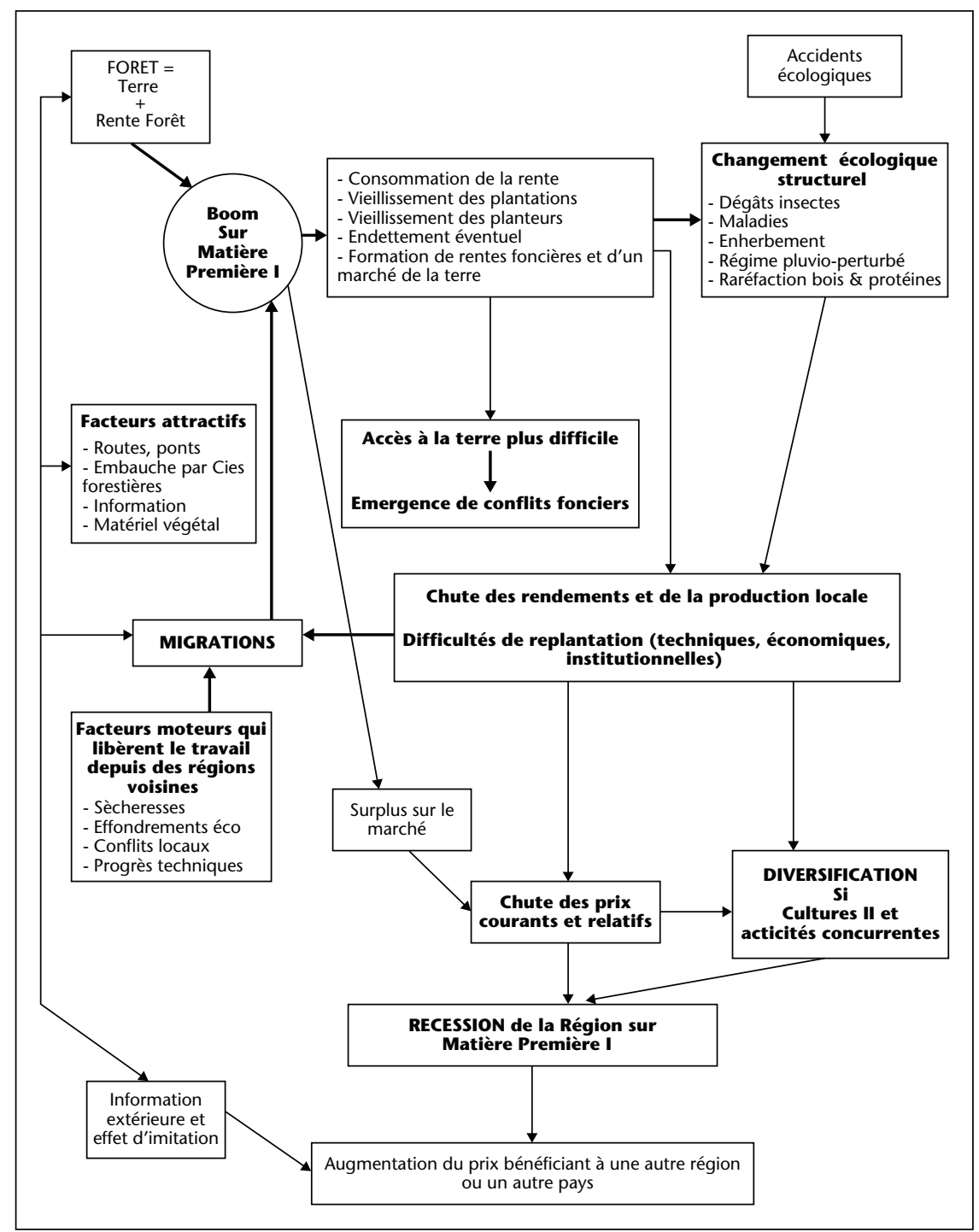

Figure 1. Cycle économique d'un produit agricole partant de la colonisation d'un milieu naturel par des migrants : modèle qualitatif. 
30 ou 40 ans, les arbres usent le milieu et s'usent eux-mêmes, baissant de rendement et d'efficacité.

\section{Usure des hommes et conjonction des cycles de vie «plantation/planteur »}

Les planteurs vieillissent avec leurs plantations, et leurs enfants partis à l'école ne sont pas toujours disponibles pour l'agriculture. Lorsque le planteur décède, les enfants sont loin et les plantations sont abandonnées et finissent par disparaître à leur tour ; ou, s'ils reviennent du vivant de leur père, les parents ne leur laissent pas toujours facilement accès aux terres; ou s'ils obtiennent une parcelle, certains ne sont pas aussi motivés que leurs parents et deviennent de simples rentiers et piètres gestionnaires d'un patrimoine déclinant.

Ou bien certains restent axés sur la même culture mais lâchent ce patrimoine et cet environnement dégradé pour partir, dans une autre région, à la recherche de forêts pour créer de nouvelles plantations. Ils participent alors à un grand mécanisme de déplacement des foyers de production d'une région à l'autre.

Au plan foncier, l'arrivée massive de migrants et la démographie naturelle densifient rapidement un milieu initialement «vierge ». Au terme d'une génération, différentes règles d'usage se construisent, différents arrangements institutionnels, en relation avec l'histoire locale, précoloniale, coloniale, postcoloniale. Malgré le caractère souvent socialement innovant de ces règles, les conflits sont inévitables. L'engouement pour la culture raréfie les terres, en augmente le coût d'accès, génère des conflits fonciers entre autochtones et migrants et au sein des familles autochtones. Globalement, une rente foncière tend à se mettre en place, pouvant apparaître comme une barrière supplémentaire à ceux qui ne possèdent pas la terre. Ainsi, la crise ivoirienne peut apparaître comme une expression partielle de ces conflits liés aux migrations cacaoyères [14-16].

Les changements démographiques et sociaux sont au cœur des retards d'investissements dans un nouveau cycle de plantations. Si des fils reviennent pour récupérer l'héritage, faute de trouver des emplois en ville, ils se trouvent plongés dans des conflits fonciers soit avec les migrants auxquels leurs parents ont vendu la terre, soit au sein de la famille autochtone. S'il y a partage des revenus entre plusieurs membres de la famille, le fils gestionnaire tend à se désintéresser de la plantation familiale et cherche à recréer sa propre plantation, mais il est handicapé par la dégradation des terres et le manque de capital.

\section{Endettement}

Les ciseaux se resserrant entre des revenus décroissants et des charges croissantes, une des formes de récession les plus fréquentes est l'endettement, enfermant les planteurs dans une spirale dont ils ne peuvent plus se défaire. Par exemple, sur le cacao, les dangers de I'endettement chronique sont signalés dès les années 1930 [17].

\section{Usure des prix}

Ainsi, 25, 30 ou 40 ans après une phase d'essor économique, et précisément au moment où il faudrait commencer à replanter, tous les types de difficultés se combinent : écologiques, techniques et sociales. Si, en plus, le prix de la matière première s'écroule du fait de l'excès d'offre sur le marché, lui-même lié à l'excès de plantations nouvelles, toutes les conditions du déclin des vieilles régions cacaoyères sont réunies. Grâce à de nouvelles migrations vers d'autres régions ou dans d'autres pays, après quelques années d'ajustement par le cours mondial, le relais d'approvisionnement du marché mondial sera assuré. C'est le principe de déplacement des foyers de production.

À l'échelle d'un pays ou d'une région, ce principe permet d'assurer une apparente stabilité de la structure d'âge des plantations, et donc de l'offre qui peut suivre longtemps une apparente croissance linéaire. En fait, cette apparente linéarité résulte de la combinaison de déclins brutaux dans certaines régions ou microrégions, compensés par des croissances exponentielles dans d'autres. Quant à la région en déclin, elle met plusieurs années ou décennies à s'en remettre, parfois par replantation, surtout par diversification. Au moins pour une part, ce réinvestissement est entrepris par une nouvelle génération de planteurs.

\section{Replantation et diversification \\ par une nouvelle génération ou/et via un marché foncier}

Une partie des planteurs bien installés, en fin de cycle de vie, peuvent investir dans la replanta- tion et la diversification. Ils ont souvent l'avantage de contrôler la terre. Mais une majorité ne peut plus faire face à l'ensemble des contraintes. À l'échelle d'une région, pour tout un ensemble de raisons techniques (difficultés de la replantation dans un milieu écologique dégradé), économiques (manque de capital, difficultés à recapitaliser), sociales (conflits fonciers, conflits d'héritage), il faut souvent attendre plusieurs années avant qu'une phase de réinvestissement dans des replantations puisse être enclenchée, majoritairement par une seconde génération. Ce changement de génération peut s'opérer à l'intérieur de la famille, par des héritages ou cessions de plantations, soit via un marché foncier, par des personnes extérieures à la famille et au village, venant acheter les vieilles plantations pour aussitôt replanter, souvent en changeant de culture. Ainsi, en Côte-d'Ivoire, un des grands multiplicateurs d'investissement dans le cacao a été le rachat de vieilles caféières à de vieux autochtones par de jeunes Burkinabés qui développent des techniques de replantation.

Ces caractéristiques sont intégrées dans le modèle, susceptibles de s'appliquer à des degrés divers aux cultures pérennes. Qu'en est-il pour le cocotier dans le cas du Ghana? Les enquêtes conçues pour tester le modèle ont été conduites d'abord, en 2005 et 2006, dans le district de N'Zema Est puis, en 2007 et 2008, dans celui de Jomoro. L'analyse repose sur ces deux enquêtes.

\section{Application au cocotier et au cycle de vie familial}

\section{Vieillissement des planteurs}

Les planteurs âgés ont le monopole des cocotiers: de vieux cocotiers pour l'essentiel (tableau 1). Les jeunes, ayant peu accès à la terre dans leurs propres villages, ont peu accès aux cocotiers. Ils sont peut-être aussi peu enclins à y travailler, comme leurs parents I'affirment. Mais indépendamment des responsabilités de chaque génération, la notion de vieillissement des planteurs et de leur capital, la culture pérenne est donc plus

Tableau 1. Superficie en cocotiers et âge du chef de famille (Région Axim, district de Nzema, 2005).

\begin{tabular}{|lllll|}
\hline Âge des chefs de famille & & & \multicolumn{2}{l|}{ Superficie en cocotiers (en acres) } \\
\cline { 1 - 2 } \cline { 5 - 5 } \cline { 5 - 5 } & Moyenne d'âge & & Local & Hybride \\
\hline$>40$ & 61 & 14,2 & 0,8 \\
\hline$<40$ & 32 & 0,5 & 0 \\
\hline Tous & 49 & 8,9 & 0,5 \\
\hline
\end{tabular}

Sources : enquêtes des auteurs, avril 2005. 
que vérifiée: indépendamment de toute maladie du cocotier, la filière se fragilise par un manque de renouvellement des ressources humaines.

\section{Vieillissement des plantations et déplacement des foyers de production}

\section{Déplacements du sud-est vers le nord-ouest dans le district de Nzema Est}

Au moment où s'arrêtent les investissements cocotiers dans les villages décimés par la maladie du jaunissement mortel du cocotier, comme Asanta et Bobrama, en bord de mer, frappés vers 1990 (figure 2), un autre, Nkroful, à $5 \mathrm{~km}$, encore peu attaqué en 2005, maintient alors son rythme de plantation (figure 3 ). Et dans plusieurs villages encore indemnes de la maladie, à la marge de la «boucle du cocotier », comme N'Vuma (figure 4), à $10 \mathrm{~km}$, et surtout Sowodadzem (figure 5), à quelque $35 \mathrm{~km}$, on trouve encore une reprise des investissements sur le cocotier, ce qui représente un début de glissement des foyers de production, en s'éloignant de la mer.

\section{Déplacements du sud vers le nord} dans le district de Jomoro : diagnostic en 2008 À Jomoro, les cocoteraies sont encore indemnes de la maladie. Le déplacement des foyers de plantations s'opère donc par la contrainte foncière et, donc, toujours par le vieillissement des plantations et planteurs. À Half Assinie, près de la mer, et Atuabo, les planteurs sont âgés et les terres occupées par les vieux cocotiers, avec peu de possibilités de replantation et de diversification (figures 6,7). Plus à l'intérieur des terres, dans un village comme Sameneye, des planteurs plus jeunes, venus chercher des terres, peuvent simultanément planter des cocotiers et commencer ou recommencer à diversifier.

\section{Déplacements probables} vers de nouvelles provinces

Nous n'avons pas pu conduire des recherches extensives dans les régions au nord de la région Ouest, mais une brève mission dans les provinces Ashanti et Brong Ahafo semble bien montrer un nouvel essor du cocotier, en réponse à la demande en noix fraîches du nord du pays et des pays voisins. Ces premières observations sont autant de signes d'un possible déplacement des foyers de production d'une province à l'autre. Si ce mécanisme se vérifie, il ne ferait que reproduire le déplacement historique des années 1930 depuis la région de la Volta vers la région Ouest.

\section{Diminution et faiblesse des rendements}

Selon les spécialistes du cocotier, une plantation hybride adulte, conduite de façon inten-

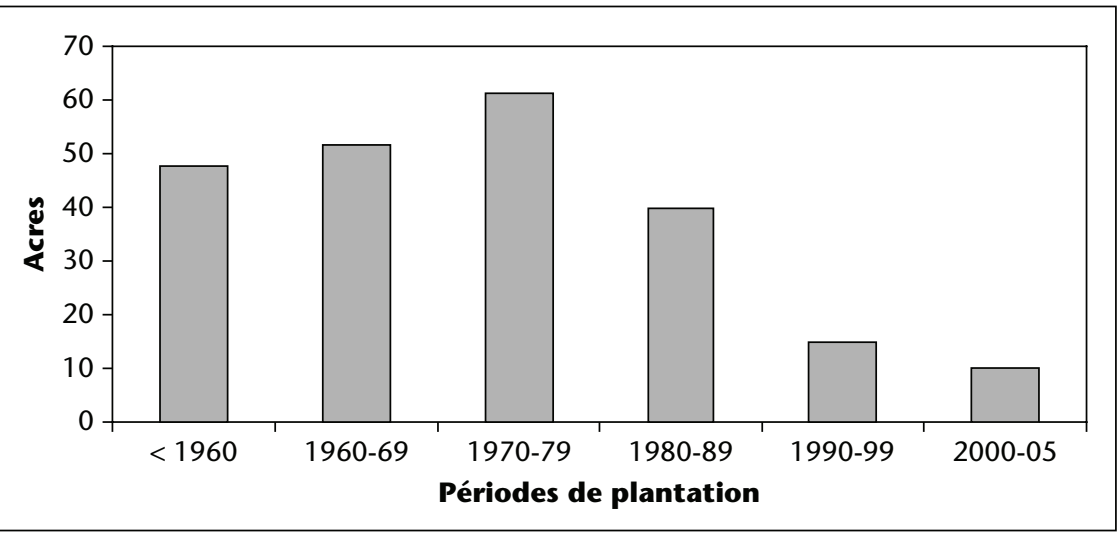

Figure 2. Structure d'âge des plantations de cocotiers à Asanta et Bobroma (zone littorale), 16 planteurs autochtones.

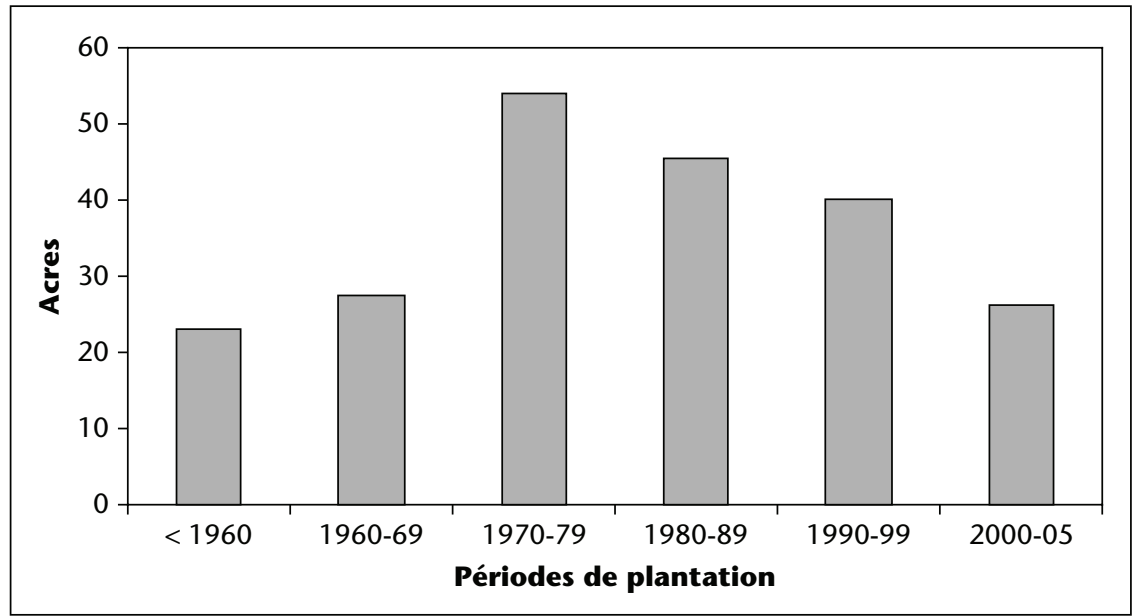

Figure 3. Structure d'âge de cocoteraies à Nkroful (5 km à la mer), 14 planteurs autochtones.

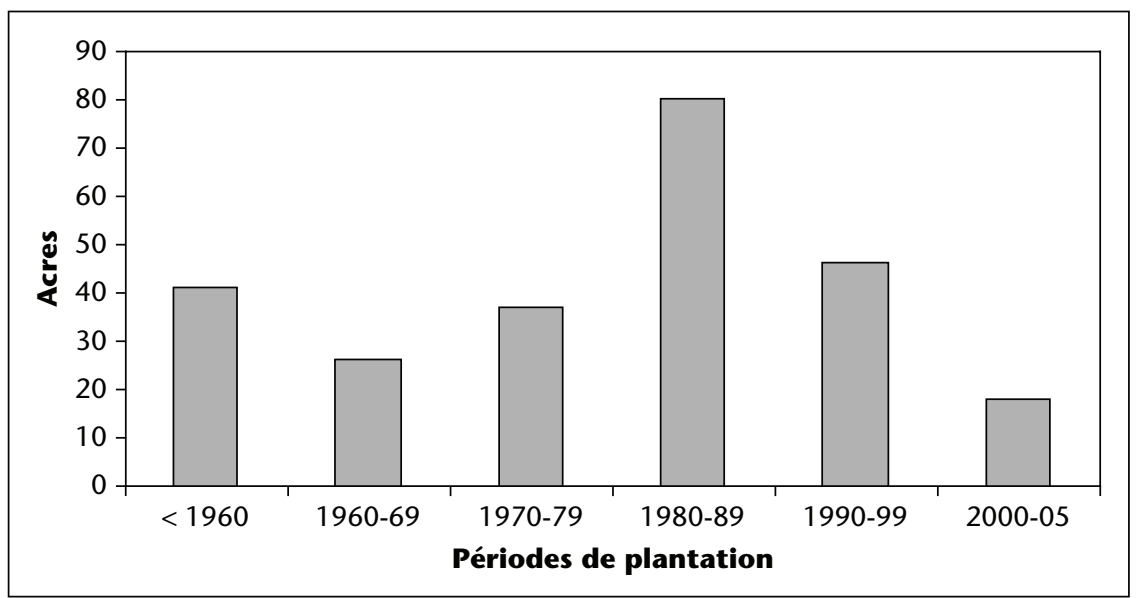

Figure 4. Structure d'âge des cocoteraies à Nvuma (à $10 \mathrm{~km}$ de la mer), 28 planteurs autochtones et migrants.

sive en Afrique de l'Ouest, avec engrais et entretien soigné, produit quelque 25000 noix. Admettons qu'en conditions paysannes, une plantation familiale pourrait en produire 20 000. Le conditionnel est obligatoire puisque ce type de plantation n'existe pas ou très peu au Ghana. Selon les planteurs enquêtés en 2008 dans la région de Jomoro, en l'absence de maladie du jaunissement mortel, la moyenne des rendements se situerait entre 


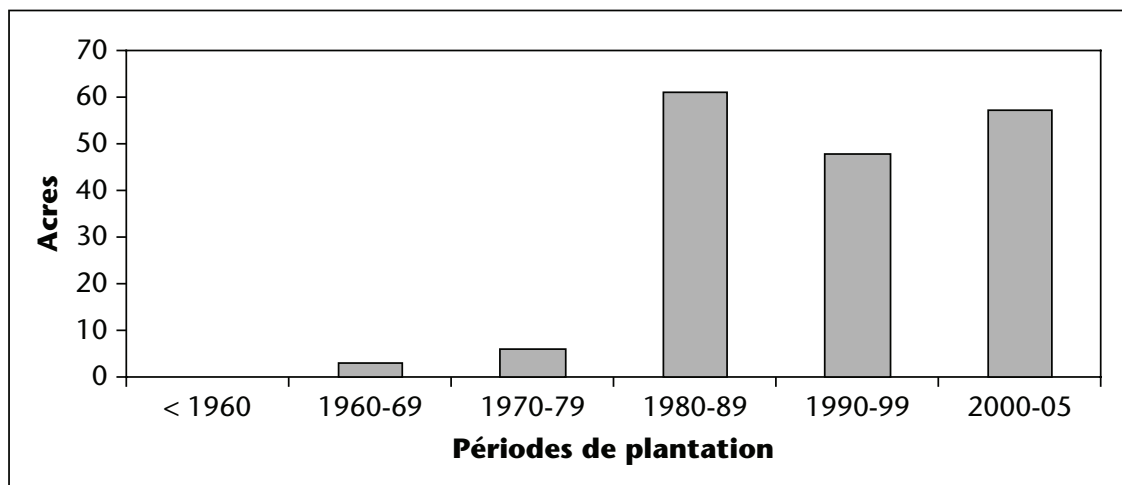

Figure 5. Structure d'âge des plantations de cocotiers à Sowodadzem (35 km de la mer), 25 planteurs autochtones.

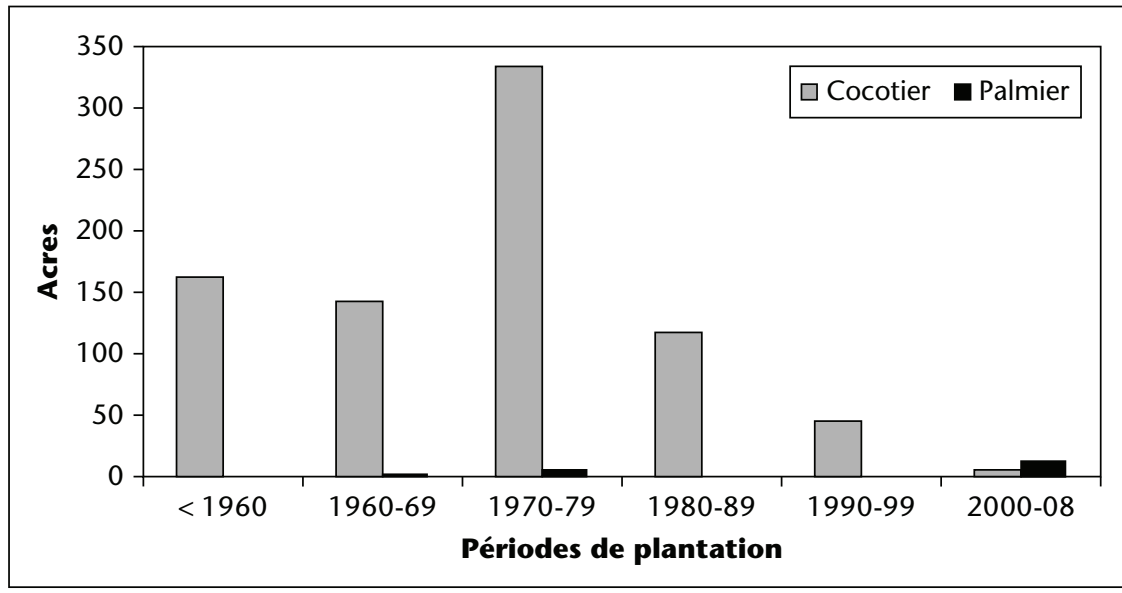

Figure 6. Structure d'âge des plantations à Half-Assinie en 2008.

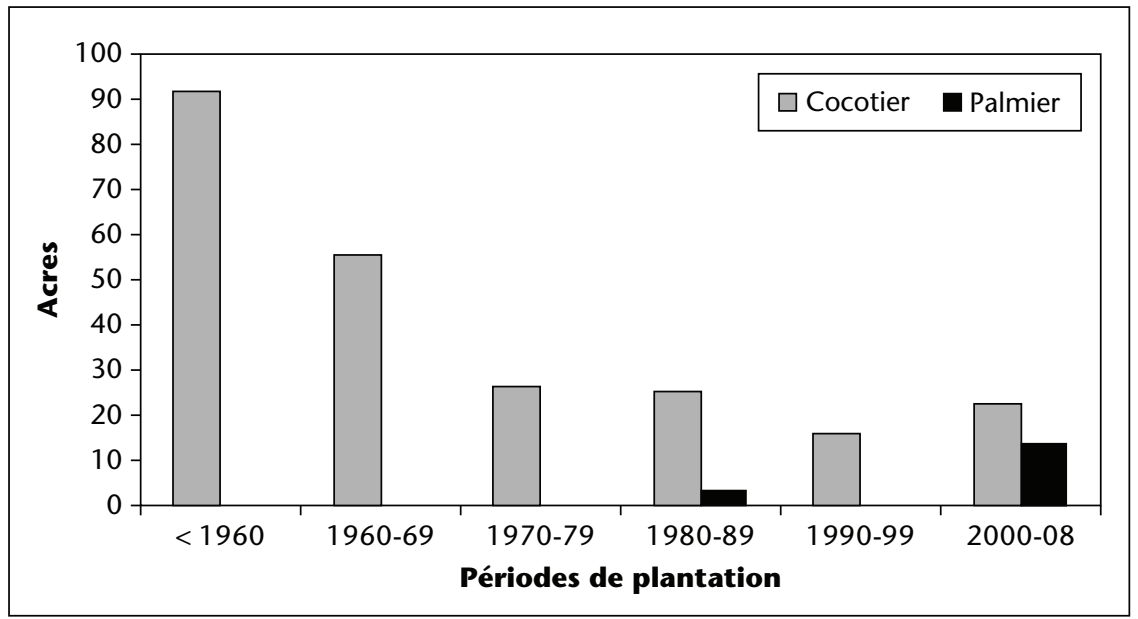

Figure 7. Structure d'âge des plantations à Jomoro, village de Atuabo, 2008, 30 planteurs.

5000 et 10000 noix, en baisse depuis plusieurs années (figure 8). La conduite est donc extensive. Le système de récolte est simplifié, réduit au strict minimum, les planteurs laissant les noix tomber d'elles-mêmes. Avec une quantité de travail très réduite et des rendements passant du moyen au faible, l'économie locale du cocotier évolue comme beaucoup de monocultures: très efficace au début pour optimiser le travail, presque rentière, mais vulnérable. C'est bien cette vulnérabilité que révèle la maladie du jaunissement mortel.

\section{D'où une stratégie de diversification hors des zones de cocotiers}

Au-delà de l'homogénéité de la structure d'âge des vergers, l'enseignement le plus manifeste de l'enquête est l'amorce de la diversification dans les années 1990, et leur plein essor dans les premières années 2000, en particulier le cacao, et dans une moindre mesure, l'hévéa (figure 9).

Pour des planteurs de cocotiers, le palmier à huile, que I'on pourrait aussi qualifier de «palmier à vin » représente aussi la diversification la plus naturelle, souvent en relais des autres. La décision la plus simple peut être aussi une stratégie d'abandon temporaire de la plantation de cocotiers.

\section{Diversification des cultures et des cycles familiaux}

À cette diversification hors des zones de cocotiers, dans les parties de terroirs villageois où les forêts secondaires et jachères sont relativement abondantes, correspond très exactement une migration de jeunes (tableau 2). Seule une minorité de ces jeunes sont fils des planteurs de cocotiers. La majorité est composée de jeunes migrants venus du pays Ashanti, du Brong Ahafo et du nord du pays.

Avec cette diversification, on retrouve donc que ce processus de rupture est quasi universel dans les économies de plantations familiales : les vieux planteurs, faute de pouvoir s'entendre avec leurs fils, cèdent la terre sous différentes formes à de jeunes migrants. Les économies de plantations restent bien familiales, mais les familles changent avec les générations et les cultures pérennes.

\section{Diversification et Abunu : contrats d'échange terre/travail}

Avec cette diversification, on retrouve également les grands modes d'accès à la terre observés en économie cacaoyère. Ici, au Ghana, notamment pour le cacaoyer, la forme d'accès à la terre la plus courante est le contrat « abunu » : le propriétaire de la terre ou supposé tel, attribue une terre. Celui la reçoit doit planter entièrement la parcelle avec la culture choisie par le propriétaire. Lorsque la plantation entre en production, les deux personnes partagent la plantation, généralement à moitié égale. Ces contrats sont équivalents au « Dibyma-Diby » au Togo et au « partager-travailler » en Côte-d'Ivoire, bagi tanah en Indonésie.

On retrouve donc là encore le modèle d'évolution : la replantation et la diversification se réalisent surtout par un changement de génération, et par une forme de transaction foncière, par une forme de marché de la terre [18]. 


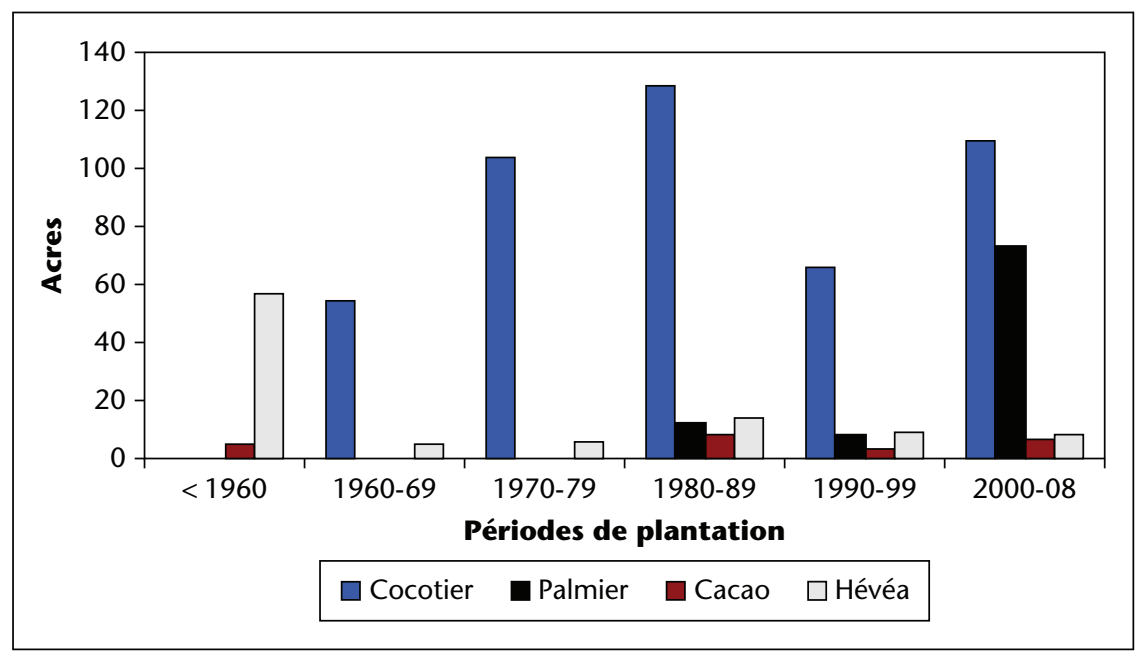

Figure 8. Structure d'âge des plantations à Jomoro, village de Sameye, 30 planteurs.

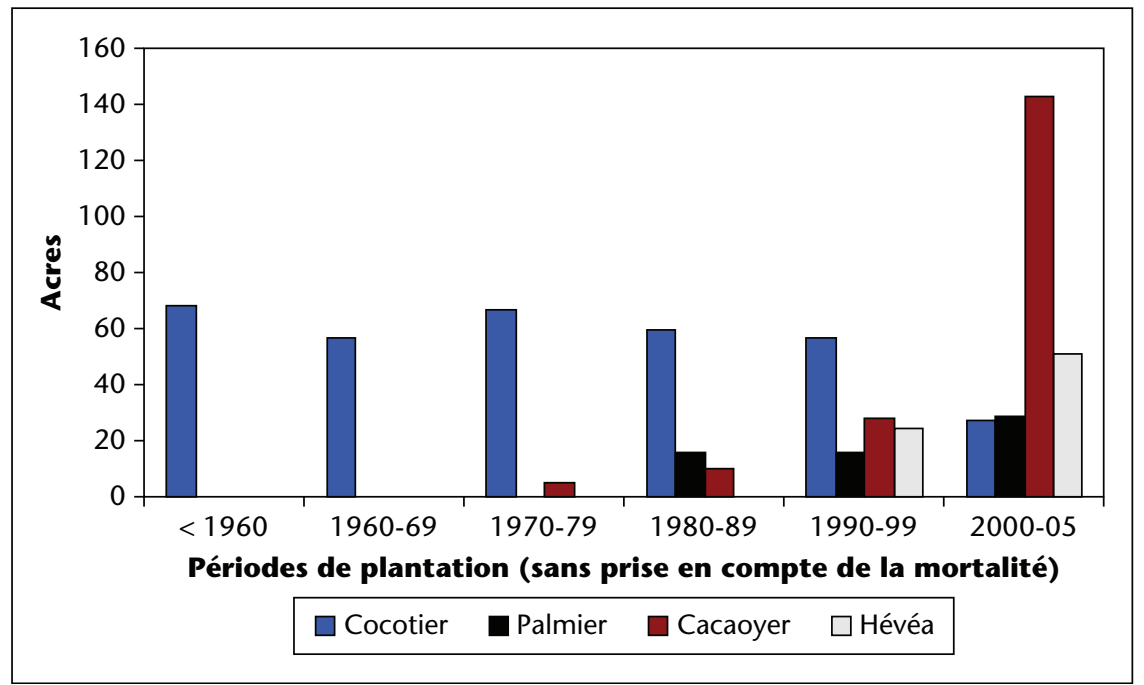

Figure 9. Investissements dans les plantations, région d'Axim (de Asanta jusqu'à Asasetre, de 0 à $20 \mathrm{~km}$ à l'intérieur des terres).

Tableau 2. Structure des exploitations et âge du chef de famille (Axim, district de Nzema, 2005).

\begin{tabular}{|c|c|c|c|c|c|c|c|}
\hline \multicolumn{2}{|c|}{ Âge des planteurs } & \multicolumn{5}{|c|}{ Surface des plantations (acres) } & \multirow{3}{*}{$\begin{array}{l}\text { Dépendance/ } \\
\text { cocotier (\%) }\end{array}$} \\
\hline & \multirow[b]{2}{*}{ Moyenne } & \multicolumn{2}{|c|}{ Cocotier } & \multirow[t]{2}{*}{ Palmier } & \multirow[t]{2}{*}{ Cacaoyer } & \multirow[t]{2}{*}{ Hévéa } & \\
\hline & & Local & Hybride & & & & \\
\hline$>40$ & 61 & 14,2 & 0,8 & 1,3 & 6,5 & 4,0 & 56 \\
\hline$<40$ & 32 & 0,5 & 0 & 2,8 & 5,6 & 2,6 & 4 \\
\hline Tous & 49 & 8,9 & 0,5 & 1,9 & 6,2 & 3,4 & 44 \\
\hline
\end{tabular}

Sources : enquêtes des auteurs, 2005.

\section{Hausse de prix de la noix de coco}

Pour éliminer le facteur spécifique de la maladie du jaunissement mortel, ces enquêtes sont faites dans la région de Jomoro, le cœur de la «boucle du cocotier», encore indemne de la maladie. C'est d'ailleurs, pour l'instant, surtout dans cette région de production encore significative que la hausse de prix s'opère. Elle est, en effet, principalement initiée par l'arrivée de nouveaux acheteurs, nigérians, lesquels recherchent à la fois une production encore importante et des noix de cocotiers locaux, plus adaptées que les hybrides au commerce entre pays de la sous-région car supportant mieux plusieurs semaines de stockage et de transport. Ces acheteurs nigérians sont donc venus développer une nouvelle filière de noix mâtures, exportées vers le Nigeria, venant s'ajouter à la filière historique de fabrication d'huile de coco, a priori en concurrence de cette filière. De fait, dans les quelques récents documents qui existent sur le cocotier au Ghana, ces deux filières sont souvent présentées comme alternatives et concurrentes. On va voir qu'elles sont complémentaires.

\section{Réalité et nuance de l'augmentation du prix à l'initiative des acheteurs}

Prix courant et prix constant

Les acheteurs nigérians, profitant $d^{\prime}$ une rente générée par le prix élevé des noix au Nigeria, et par le taux de change, augmentent régulièrement leurs prix à l'achat, en partie, sous l'effet de la concurrence qu'ils exercent entre eux (figure 10). Tous les prix donnés ici sont exprimés en "Ghana cedis ", échangé autour de 0,66 euro au tournant des années 2007 et 2008 ( 1 euro = 1,45 à 1,55 Ghana cedi).

Toutefois, une première nuance consiste à rappeler le degré d'inflation relativement élevé ces dernières années au Ghana. En prix constants, ici déflatés par l'indice de consommation moyen au Ghana, cette augmentation est quelque peu « écrasée » (figure 11).

Néanmoins, ce prix offert par les acheteurs nigérians représente une augmentation réelle par rapport aux années antérieures à leur arrivée. En trois ans, le prix réel double..., ou semble doubler, car les services rendus ne sont pas les mêmes.

\section{Prix chez l'acheteur et prix bord champ}

Derrière les prix affichés se cache une inégalité de service, pas toujours transparente. En fait, le prix élevé offert par les acheteurs nigérians est un prix rendu magasin, alors que le prix « un achat comptant » proposé par la filière « huile de coco » est un prix bord champ, économisant également l'opération de débourrage des noix, qui est alors fait par l'atelier. II reste une 


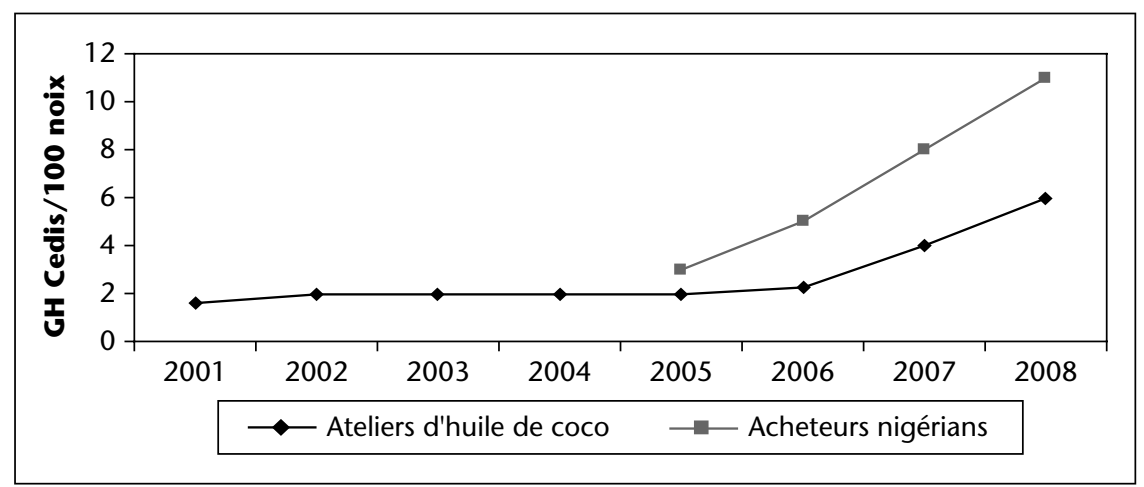

Figure 10. Prix courants des noix de coco offerts par les acheteurs, 2001 à mai 2008 (paiement comptant).

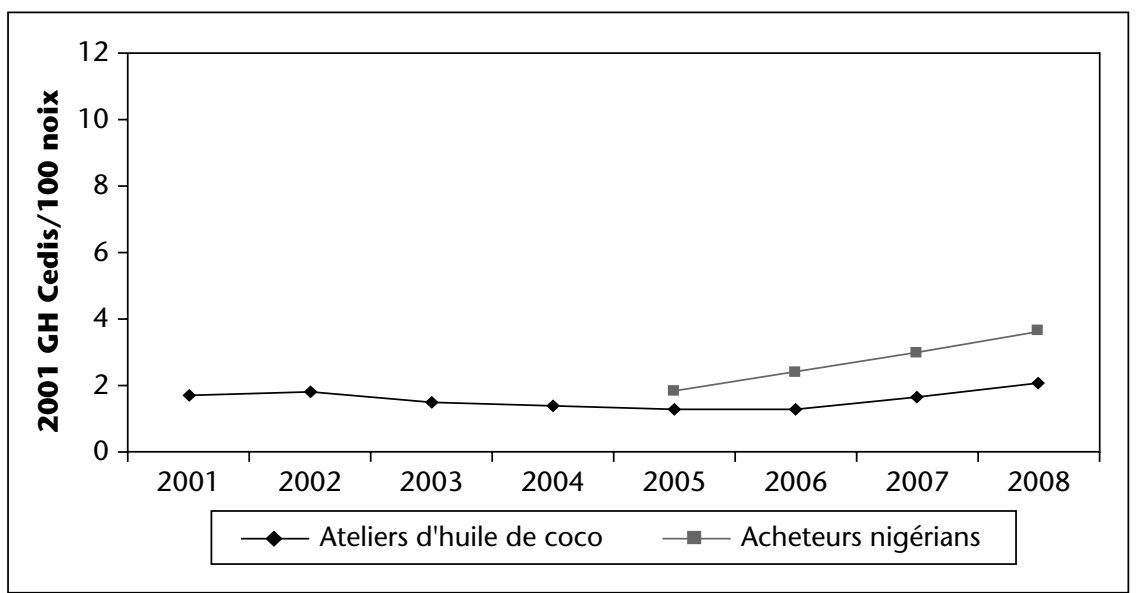

Figure 11. Prix constants des noix de coco offert par les acheteurs, 2001 à mai 2008 (paiement comptant).

Tableau 3. Prix courants bord champ sur les deux filières (prix pour achats comptants).

\begin{tabular}{|lll|}
\hline & $\begin{array}{l}\text { Atelier de transformation } \\
\text { en huile }= \\
\text { prix bord champ }\end{array}$ & $\begin{array}{l}\text { Vente directe } \\
\text { aux Nigérians }= \\
\text { prix rendu magasin }\end{array}$ \\
\hline Prix payé au producteur & 4 à 6 & 8 à 11 \\
\hline Débourrage de la noix & 0 & 0,5 à 0,6 \\
\hline Transport du champ au village & 0 & 0,5 à 1,5 \\
\hline Total coûts & 0 & 1,0 à 2,1 \\
\hline Prix bord champ en paiement comptant & 4 à 6 & 5,9 à 10,0 \\
\hline
\end{tabular}

Sources : enquête des auteurs, 2007 et 2008.

différence en faveur des acheteurs nigérians, mais surtout sensible depuis 2007, et impliquant que le planteur accepte des tâches auxquelles il n'est pas habitué (tableau 3).

En résumé, en 2005 et 2006, avec un prix de 5 à 6 Chana cedis pour 100 noix, la hausse du prix bord champ ou de son équivalent était encore limitée. À partir de 2007 et d'un prix à 7 cedis, elle devient plus significative et permet de tester la capacité de réponse au prix des planteurs de Jomoro. fiter de la hausse de prix. Ce sont de nouvelles régions ou autres pays qui vont en profiter. Estce le cas pour ce secteur cocotier du Chana? Quelle réponse des planteurs dans la «vieille zone de production »?

Cette capacité de réponse est testée par enquêtes auprès de 90 planteurs de Jomoro, sur plusieurs critères : le niveau de l'entretien des parcelles, celui de l'adoption des engrais et les décisions d'investir dans des replantations et de nouvelles plantations, en local et en hybrides.

\section{Entretien}

Selon les déclarations des planteurs, la hausse de prix de la noix de coco ne génère aucune incitation à mieux nettoyer les plantations de cocotiers. La moyenne des nettoyages était de 1,3 avant leur arrivée en 2005 et de 1,4 en 2007 : pas de différence significative.

Au contraire, les enquêtes mettent en exergue le maintien d'un quart des plantations en conduite très extensive, avec seulement un nettoyage tous les trois à cinq ans. II s'agit alors d'un simple éclaircissement pour accéder aux cocotiers et maintenir un cercle à peu près propre autour de chaque cocotier, afin de limiter la perte des noix de coco. Rappelons que les planteurs laissent les noix tomber d'elles-mêmes et doivent les retrouver dans la végétation arbustive qui se développe sous les cocotiers, à hauteur d'homme, voire au-delà.

\section{Adoption et application de l'engrais}

Les acheteurs nigérians n'ont rigoureusement aucun impact sur les achats d'engrais. De 2005-2006 à 2007-2008, seulement $2 \%$ des planteurs en ont utilisé. II ne s'agit pas prioritairement d'un problème d'information. Entre 1982 et 2004, le pourcentage des planteurs ayant déclaré une application d'engrais s'élève à $24 \%$, principalement grâce à des subventions, des engrais livrés gratuitement dans les années 1990. Au-delà des questions de prix, une des principales contraintes ressenties par les planteurs est celui du transport de l'engrais de la maison à la plantation.

\section{Replantations et plantations}

Les décisions d'investissement dans la replantation et de nouvelles plantations restent insensibles à la hausse de prix. Du moins, les investissements plafonnent à des taux insignifiants (tableau 5).

Les résultats d'enquête obtenus à Jomoro semblent donc bien correspondre au modèle : les planteurs d'une vieille région de production, en quasi-monoculture, ne semblent pas en mesure de réagir à la hausse de prix par des investissements. Mais s'agit-il bien des mêmes déterminants? 
Tableau 4. Nombre d'entretiens annuels dans les plantations.

\begin{tabular}{|lll|}
\hline & $\begin{array}{l}\text { Nombre de } \\
\text { planteurs }\end{array}$ & Pourcentage \\
\hline 0,2 à 0,3 & 20 & 23 \\
\hline 1 & 35 & 40 \\
\hline 2 & 18 & 21 \\
\hline 3 & 14 & 14 \\
\hline Total & 87 & 100 \\
\hline
\end{tabular}

\section{Retour simultané au modèle et au réel}

Avec Jomoro, on retrouve bien le processus décrit dans le modèle, en particulier les fondamentaux tels que la saturation foncière, le vieillissement des plantations et des planteurs, certains conflits sociaux, le crédit usurier se développant avec la baisse des revenus et la saisonnalité des revenus en monoculture. Tous ces facteurs dissuadent l'investissement. Mais ce secteur cocotier a tellement amplifié le facteur « endettement » que nous commençons par celui-ci.

\section{Endettement chronique : valeur ajoutée captée par les intermédiaires}

L'endettement, associé à ce fameux squeeze entre des revenus décroissants et des charges croissantes, est bien un des mécanismes classiques de la récession dans le modèle. Mais, pour ce secteur cocotier à Jomoro, il connaît un effet particulièrement dévastateur, pourtant peu évoqué dans la littérature.

Ainsi, en point de départ, et malgré le "modèle ", notre hypothèse était que la hausse des prix induite par l'arrivée des Nigérians allait permettre aux planteurs de se dégager progressivement. Au moins à partir des observations prolongées jusqu'au début de 2008 il n'en est rien.

Selon l'enquête conduite à Jomoro, seulement $26 \%$ des planteurs ont accès aux acheteurs nigérians et à leurs prix plus élevés que ceux de la filière "huile de coco». La majorité, $74 \%$ des planteurs, n'y ont pas accès. Les raisons sont avant tout l'endettement et le besoin de crédit (tableau 6).

Non seulement une majorité de planteurs reste prisonniers de leurs crédits, mais ils anticipent en avoir besoin. Ils se privent des prix plus élevés des acheteurs nigérians, lesquels ne fournissent pas de crédit. Les planteurs continuent de vendre

Tableau 5. Surfaces de plantations et replantations de cocotiers.

\begin{tabular}{|lll|}
\hline & $\mathbf{2 0 0 1 - 2 0 0 4}$ (acres) & $\mathbf{2 0 0 5 - 2 0 0 8}$ (acres) \\
\hline Replantations (tout en local) & 0,14 & 0,08 \\
\hline Plantations (en local) & 0,07 & 0,05 \\
\hline Plantations (en hybrides) & 0,05 & 0,01 \\
\hline
\end{tabular}

Sources : enquête des auteurs, 2008.

Tableau 6. Raisons évoquées par les planteurs pour ne pas vendre aux acheteurs nigérians.

\begin{tabular}{|lll|}
\hline & $\begin{array}{l}\text { Nombre } \\
\text { de plantes }\end{array}$ & Pourcentage \\
\hline « Prisonnier » de l'endettement auprès de son acheteur traditionnel & 34 & 53 \\
\hline Les Nigérians ne fournissent pas de crédit & 8 & 13 \\
\hline $\begin{array}{l}\text { Ne veut pas prendre en charge le coût additionnel du débourrage } \\
\text { des noix et le transport }\end{array}$ & 13 & 20 \\
\hline Pas de confiance dans un acheteur étranger & 8 & 13 \\
\hline $\begin{array}{l}\text { Lui-même acheteur pour la transformation en huile, } \\
\text { ne veut pas revendre au Nigérian }\end{array}$ & 1 & 2 \\
\hline Total & 64 & 100 \\
\hline
\end{tabular}

Sources : enquête des auteurs, 2008.

Tableau 7. Prix effectivement obtenus par les planteurs fin 2007 compte tenu de l'endettement.

\begin{tabular}{|lccl|}
\hline $\begin{array}{l}\text { Prix réellement touché } \\
\text { par le planteur (Ghana } \\
\text { cedis) pour } \mathbf{1 0 0} \text { noix }\end{array}$ & $\begin{array}{l}\text { Nombre de } \\
\text { planteurs }\end{array}$ & Pourcentage & Commentaires \\
\hline 2 & 5 & 74 & $\begin{array}{l}74 \% \text { n'ont pas eux-mêmes accès aux } \\
\text { acheteurs nigérians, du moins pas à leurs prix }\end{array}$ \\
3 & 39 & & \\
4 & 18 & & $14 \%$ bénéficient d'un prix intermédiaire, \\
5 & 2 & 14 & via des intermédiaires \\
\hline 6 & 2 & $12 \%$ ont un accès direct aux acheteurs \\
7 & 10 & & nigérians et à leurs prix \\
\hline 8 & 2 & & \\
9 & 7 & 100 & \\
10 & 1 & &
\end{tabular}

Sources : enquête des auteurs, 2008.

aux acheteurs/transformateurs ghanéens qui seront toujours prêts à leur concéder un crédit.

L'enquête à Jomoro révèle donc que l'endettement écrase les prix. Ce qui est offert au planteur en guise de prix associé à un crédit n'a plus rien à voir avec les prix affichés au comptant (tableau 7).

De tels chiffres et de tels résultats, émerge une question inévitable : comment les acheteurs nigérians s'approvisionnent-ils en noix de coco si seulement un quart des planteurs leur vendent des noix ? Le premier élément de réponse est bien sûr relatif au rôle des intermédiaires.

Pour une grande partie des $74 \%$ des planteurs devant se contenter des prix de 3 à 4 Ghana cedis pour 100 noix, exceptionnellement 2 Ghana cedis, leurs noix de coco arrivent bel et bien dans les magasins des acheteurs nigérians. Mais ceux qui touchent leurs prix autour de 7 Ghana cedis et réalisent la plus-value sont les intermédiaires. 
Ces derniers ont construit leur domination du marché dans des conditions "favorables": acheteurs nigérians pressés qui ne connaissent pas le pays et les paysans ayant besoin d'intermédiaires, vieux paysans ayant besoin $d^{\prime}$ aide pour le débourrage des noix et surtout le transport. Mais la raison essentielle de cette emprise sur le marché est l'endettement chronique et l'anticipation par les paysans de leur besoin en crédit. Ils paient très cher la sécurité de pouvoir emprunter à tout moment.

Qui sont ces intermédiaires? Nous n'avons pas conduit d'enquêtes spécifiques sur eux, mais une grande partie d'entre eux se compose des transformateurs (et transformatrices) en huile de coco. Ce résultat est un des plus inattendus de l'enquête. Certains transformateurs, principaux opérateurs de la filière avant l'arrivée des Nigérians, se sont fort bien adaptés à la concurrence des nouveaux acheteurs nigérians : en la contournant, en devenant leurs intermédiaires. De fait, ils avaient déjà les clients, et ils les « tenaient » par l'endettement. En plus, les ateliers de transformation en huile peuvent maintenir une partie de leur activité ; ils peuvent valoriser en huile les plus petites noix souvent rejetées par les acheteurs nigérians.

\section{Conflits de génération, hausse des prix et vols de noix}

Le discours des vieux planteurs désabusés se résume par leur complainte envers les jeunes qui volent les noix. II s'agit souvent des jeunes du village, parfois de la même famille. Même s'il convient de prendre les chiffres avec la plus grande précaution, la moyenne des estimations de planteurs donne $20 \%$ de perte de noix par vol avant l'arrivée des Nigérians et $67 \%$ en 2007.

Même si les discours et les chiffres sont exagérés, on a bien ici un processus d'augmentation classique des vols avec la hausse des prix, connu dans bien des économies. Il est ici accentué dans un contexte de conflit des générations, également très fréquent dans les économies familiales de plantation à base de cacaoyers, de caféiers ou de palmiers: les « vieux » contrôlant la terre et les plantations face aux « jeunes » refusant de travailler pour leurs parents, pour peu ou pas d'argent.

Dans un tel contexte, de vieillissement des plantations, de faible entretien et de vols, la hausse de prix est presque contre-productive du point de vue des planteurs, dont le revenu moyen augmenterait finalement trois fois moins que le prix effectivement perçu (tableau 8). Compte tenu de l'inflation, cela signifie finalement un pouvoir d'achat très faible, ayant très peu progressé, voire diminué.

Tableau 8. Estimation des prix et revenus des planteurs de cocotiers en 2004 et 2007.

\begin{tabular}{|lrrrl|}
\hline & $\mathbf{2 0 0 4}$ & $\mathbf{2 0 0 7}$ & Augmentation & Pourcentage \\
\hline Prix pour 100 noix (Ghana cedis) & 2,6 & 4,3 & 1,7 & 67 \\
\hline $\begin{array}{l}\text { Estimation du revenu courant pour trois mois } \\
\text { (Ghana cedis) }\end{array}$ & 241 & 305 & 63 & 26 \\
\hline
\end{tabular}

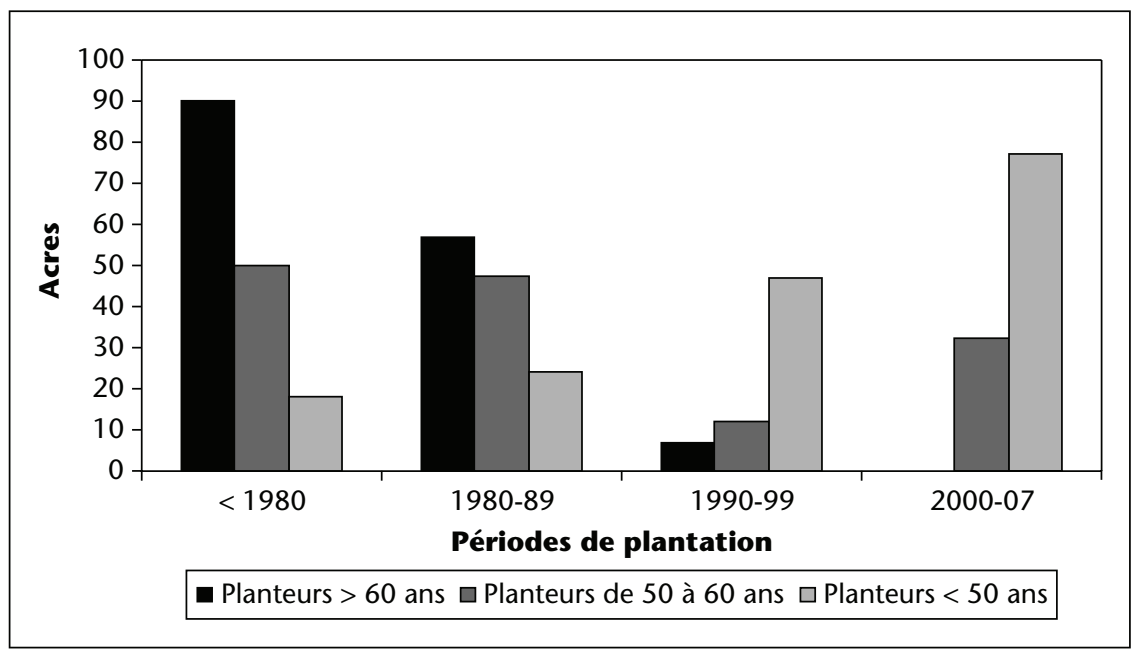

Figure 12. Structure d'âge des plantations à Sameneye (Jomoro) selon les classes d'âge des planteurs.

\section{Endettement et vols de famille}

Le vol des noix dans les plantations familiales est un problème réel et sérieux, l'expression d'un blocage de la société locale dans une économie en difficulté. Mais sans que nous puissions le démontrer ici, on peut quand même faire l'hypothèse d'accords occasionnels entre pères et fils. Un père endetté peut sans doute « tolérer » un vol au sein de la famille, qui va permettre d'échapper provisoirement au remboursement et bénéficier d'un prix plus élevé. Que le vol soit réel ou déguisé, il est d'ailleurs probable qu'une part non négligeable des approvisionnements des acheteurs nigérians vienne de ce circuit.

\section{Fondamentaux : manque de terre, vieillissement des planteurs, manque d'énergie}

Le problème de vieillissement des plantations mais aussi des planteurs a déjà été montré par les enquêtes de 2005 dans le district de Nzema Est (figure 1 et tableaux 2-4). II se confirme ici avec les enquêtes de 2007-2008 à Jomoro, montrant une moyenne d'âge élevée pour des chefs d'exploitation (tableau 9).

Le village de Sameneye est plus dynamique que les autres en termes de plantations nouvelles, notamment de cocotiers locaux, en partie,
Tableau 9. Âge des chefs d'exploitation à Jomoro.

\begin{tabular}{|ll|}
\hline & Âge moyen \\
\hline Half-Assinie & 63 \\
\hline Atuabo & 57 \\
\hline Sameneye & 55 \\
\hline Ensemble sur les trois sites & 58 \\
\hline
\end{tabular}

Sources : enquête des auteurs, 2008.

parce que l'espace n'y est pas saturé, mais aussi parce la proportion de jeunes planteurs $y$ est un peu plus forte. Ainsi, au sein du village de Sameneye, et malgré les effets des héritages, la symétrie entre les exploitations des planteurs les plus âgés et les plus jeunes est exemplaire de cette conjonction entre cycle de vie du planteur et de la plantation (figure 12). C'est la base du modèle.

\section{Conclusion}

Au fil des décennies, l'économie locale à base de cocotiers avait construit un système extensif mais productif, peu consommateur en travail, adapté au vieillissement de la population, devenu quasi-rentier, de faible rente, mais ren- 
tier quand même. Le système s'est donc fragilisé. De fait, même dans les régions indemnes de la maladie, une hausse de prix, même importante, ne suffit plus à relancer les investissements et la production : un dilemme en tout point conforme au modèle proposé, construit empiriquement par analyse comparée des économies cacaoyères. Le modèle s'applique relativement bien au cas du cocotier au Ghana.

On retrouve donc ce paradoxe très classique dans les économies de plantation, entre une demande croissante, ici surtout à l'échelle du marché de l'Afrique de l'Ouest, et l'offre du Ghana, pour l'instant en déclin. L'ajustement entre l'offre et la demande se fera peut-être par des déplacements des foyers de production, vers le nord des districts de Nzema et Jomoro, et probablement aussi vers le Brong Ahafo et la province Ashanti. Tant que la maladie du jaunissement mortel ne se développe pas en Côte$d^{\prime}$ Ivoire, ce pays bénéficiera aussi de la relance de la consommation au Nigeria et d'autres pays africains. C'est d'ailleurs déjà le cas, puisque I'agriculture familiale du sud de Bonoua reprend activement des investissements dans les plantations de cocotiers locaux [19].

En attendant, quelles sont les conséquences pour les planteurs de la région Ouest du Ghana, notamment des districts de Nzema et Jomoro ? Dans les villages dévastés par la maladie, la question de la hausse de prix est dépassée. Non seulement les plantations disparaissent, mais les planteurs aussi. Sans le démontrer ici, beaucoup de planteurs décèdent quelques mois ou quelques années après leurs plantations. C'est un des tristes aspects de la conjonction des cycles de vie des planteurs et de leurs plantations. Eux et leurs familles n'ont jamais reçu aucune aide.

Dans les régions encore indemnes de la maladie, on a donc vérifié que la hausse de prix n'était pas suffisante. La tentative d'aide, de 1999-2003, sous forme de projet de replantation de cocotier hybride, allait dans la bonne direction. Sans le démontrer ici, il fut un échec relatif pour plusieurs raisons :

- il reposait sur un seul hybride, s'avérant d'une tolérance douteuse, du moins quand la maladie est dans sa phase la plus active (même plus sensible que le "grand Ouest Africain », selon certains planteurs). Ne fallait-il pas entamer dès le début une recherche participative avec au moins deux ou trois hybrides à l'essai ? - les planteurs bénéficiaires du projet ont été mal sélectionnés : trop d'absentéistes, trop de planteurs très âgés, et les planteurs ont été peu informés des risques et des exigences de I'hybride en engrais et entretien ;

- le problème, difficile mais incontournable de l'endettement et du crédit, n'a pas été compris ou, du moins, pas pris en compte par le projet.
Il en va de même pour le problème du vieillissement des planteurs et du conflit des générations.

Mais le projet a aussi apporté des éléments positifs, peu mis en évidence. Ainsi, le matériel végétal n'est peut-être pas parfait, mais le projet a contribué à le faire connaître et à lancer sa diffusion dans d'autres provinces comme Ashanti et Brong Ahafo. Pour l'instant, les hybrides ne satisfont pas la demande des Nigérians, mais ils ont un grand potentiel comme noix fraîches demandées par le nord du Ghana et les pays limitrophes. Si ce succès imprévu du projet se confirme, ce serait un point commun de plus avec le modèle et bien des économies cacaoyères, à commencer par celle du Ghana : quand la recherche se penche sur un problème de maladie d'une culture pérenne (le swoolen shoot pour le cacao) et introduit un nouveau matériel végétal (hautamazonien dans le cas du cacao au Ghana), le résultat n'est pas celui attendu : au lieu de favoriser la replantation dans les zones touchées, il favorise en priorité l'expansion vers de nouvelles régions (en l'occurrence le Brong Ahafo puis la région Ouest).

En recommandation pour ces régions touchées par la maladie du jaunissement mortel du cocotier, les quelques lignes directrices sont de ne pas laisser sans aide des milliers de familles, de prolonger et d'accentuer les tests d'hybrides avec les planteurs les plus motivés, d'aborder le problème clef de l'endettement et du crédit.

Au final, cette histoire de cocotier est bien un cas d'école, démontrant les effets négatifs d'une monoculture sur le long terme, démontrant a contrario l'intérêt d'une diversification des cultures. C'est une des raisons du succès du projet hévéa dans la région, apportant une aide significative aux villages qui ont pu en bénéficier. Le besoin de diversification est sans doute le principal enseignement de cette crise, comme devraient l'être toutes les crises, écologiques ou économiques, venant frapper, un jour, une monoculture.

Remerciements. Nos remerciements vont naturellement à l'Ambassade de France, pour son intérêt et son soutien à ce projet de recherche, en commençant par Bernard Poudevigne, conseiller à l'Ambassade de 2004 à 2007, posant les bonnes questions, aidant à financer l'équipe et des étudiants. Olivier Robinet et Francine Meyer, successivement chefs du SCAC ont également soutenu activement le programme. En 2007 et 2008, la bonne coordination de Philippe Courbet, contribuant fortement au stage d'un bon duo d'étudiants et désormais collègues, a été également très appréciée. Enfin, nous n'oublions pas nos collègues du Cirad et de I'OPRI, agronomes, sélectionneurs, phytopathologistes, entomologistes, pour lesquels l'intrusion d'un économiste n'a pas toujours été facile!

\section{RÉFÉRENCES}

1. Geiger R, Lhommet JC. Devenir des exploitations agricoles face à la maladie du jaunissement mortel du cocotier. CNEARC et Cirad, Montpellier, 70 p., 2005.

2. Ruf $F$. Les déterminants écologiques de la diversification. La région d'Axim au Ghana: de la noix de coco au cacao. Rapport pour l'Ambassade de France à Accra. Cirad, Montpellier, 25 p., 2005.

3. GRET/IRAM. Evaluation of the coconut sector development project in Central and Western Regions in Ghana, first draft report. 2004.

4. Ollivier J, Bonneau X, Andoh Mensah E, Ransford A, Dery S. Evaluation of performances on cropping systems in the coconut replanting programme in the coastal region of Ghana. Cirad, Montpellier, 11 p., 2005.

5. Bene S. Coconut Marketing in Ghana. Training Workshop report. COFPMAG, Ghana, 30 p., 2006.

6. Ruf F. "French Project" contre "French Project" au Ghana? Développement et antagonismes entre « Replantation cocotier » et « Diversification hévéa ». Rapport d'études pour le ministère français des Affaires étrangères, Cirad, Montpellier, 15 p., 2006.

7. Ruf $F$. Éléments pour une théorie sur l'agriculture des régions tropicales humides. De la forêt, rente différentielle au cacaoyer, capital travail. Agron Trop 1987 ; 42 : 218-32.

8. Ruf F. Le cacao: cycles, coûts, externalités. Quelle place pour le politique? In: BenoitCattin $\mathrm{M}$, ed. Économie des politiques agricoles dans les pays en développement. Vol.3. Paris: Editions de la Revue française d'économie, 1994 : 149-98.

9. Ruf F, Ehret P. Yoddang. Smallholder cocoa in Indonesia: why a cocoa boom in Sulawesi? In : Clarence-Smith WG, ed. Cocoa pioneer fronts since 1800. The role of smallholders, planters and merchants. Londres: MacMillan, 1996 : 212-31.

10. Jarrige F. La dynamique d'offre de cacao entre marché, conditions naturelles et institutions; interprétation à partir d'une comparaison Côte$d^{\prime}$ Ivoire/Malaisie. Thèse de doctorat en sciences économiques, université de Bourgogne, 1994.

11. Touzard JM. L'économie coloniale du cacao en Amérique Centrale. Montpellier: Cirad. Coll. Repères, 1993. 
12. Petithuguenin P. Les conditions naturelles de production du cacao en Côte-d'Ivoire, au Chana et en Indonésie. Plantations Recherche Développement 1998 ; 5 : 393-411.

13. Ruf F. Déterminants sociaux et économiques de la replantation. OCL $2000 ; 2$ : 189-96.

14. Chauveau JP. How does an institution evolve? Land, politics, intergenerational relations and the institution of the tutorat. In : Kuba R, Lentz $C$, eds. Land rights and the politics of belonging in West Africa. Leiden : Brill, 2006 : 213-40.
15. Colin JP, Ayouz M. The development of a land market? Insights from Côte-d'Ivoire. Land Econ $2006 ; 2$ : 404-23.

16. Losch B, Mesplé-Somps S, Chauveau JP, Conta$\min B$. Le processus de libéralisation et la crise ivoirienne. Cirad et ministère des Affaires étrangères, Paris et Montpellier, 31 p., 2003.

17. Beckett WH. Akokoaso. A survey of a Gold-Coast Village. London : The London School of Economics, Monographs on Social Anthropology, 1944 (95 p.).
18. Ruf F. Yoddang. From migration to motorisation; how market liberalisation has benefitted coffee farmers? In : Gerard F, Ruf F, eds. Agriculture in crisis: people, commodities and natural resources in Indonesia, 1996-2000. UK : Curzon Press, 2001 : 197-236.

19. Ruf $F$. Éléments d'une stratégie d'amélioration du peuplement végétal des plantations pérennes paysannes en zone forestière de Côted'Ivoire. Vol 2 : La demande en matériel végétal et son utilisation par les planteurs. BDPA et Cirad, Montpellier, 220 p., 2007. 